\section{Nota corretiva}

PACE, R. 2012: Biodiversità delle Aleocharinae della Cina: Hygronomini e Oxypodini (Coleoptera, Staphylinidae). - Beiträge zur Entomologie 62 (1), 125-163, 136 figg.

Su segnalazione del Dr Eckhard Groll specializzato d'informazione in Entomologia della redazione di "Contributions to Entomology - Beiträge zur Entomologie", Müncheberg, Germania, che ringrazio, correggo qui l'omonimia seguente e propongo il seguente nome nuovo:

\section{Trichoglossina grolli nomen novum per}

Trichoglossina tronqueti PACE, 2012: 148

nec Trichoglossina tronqueti (PACE, 1987), olim Hygropetrophila tronqueti PACE, 1987: 417; PACE, 2012: 143

\section{Correction note}

PACE, R. 2012: Biodiversità delle Aleocharinae della Cina: Hygronomini e Oxypodini (Coleoptera, Staphylinidae). - Beiträge zur Entomologie 62 (1), 125-163, 136 figg.

I thank Dr. Eckhard Groll, entomology information center and editor of „Contributions to Entomology Beiträge zur Entomologie“ Muencheberg, Germany, for drawing my attention to a secondary homonym and propose hereby the following new name:

Trichoglossina grolli nomen novum for

Trichoglossina tronqueti PACE, 2012: 148

and not Trichoglossina tronqueti (PACE, 1987), formerly Hygropetrophila tronqueti PACE, 1987: 417; PACE, 2012: 143

\section{Korrektur}

PACE, R. 2012: Biodiversità delle Aleocharinae della Cina: Hygronomini e Oxypodini (Coleoptera, Staphylinidae). - Beiträge zur Entomologie 62 (1), 125-163, 136 figg.

Ich danke Dr. Eckhard Groll, Informationszentrum Entomologie und Redaktion der „Contributions to Entomology - Beiträge zur Entomologie“ Müncheberg, Deutschland, für den Hinweis auf ein sekundäres Homonym und schlage hiermit folgenden neuen Namen vor:

Trichoglossina grolli nomen novum für

Trichoglossina tronqueti PACE, 2012: 148

und nicht Trichoglossina tronqueti (PACE, 1987), ehemals Hygropetrophila tronqueti PACE, 1987, 417; PACE, 2012: 143

\section{Bibliografia / References / Literatur}

PACE, R 1987: Aleocharinae dell'Himalaya raccolte da Marc Tronquet e Georges Ledoux (Coleoptera Staphylinidae) (LXXVIII Contributo alla conoscenza delle Aleocharinae). - Bollettino del Museo civico di Storia naturale di Verona 14: 403-419, 53 figg.

PACE, R. 2012: Biodiversità delle Aleocharinae della Cina: Hygronomini e Oxypodini (Coleoptera, Staphylinidae). - Beiträge zur Entomologie 62 (1): 125-163, 136 figg. 